\title{
Cooking classes as a method of improving food engagement in a student population: a pilot study
}

\author{
N. O’Kane ${ }^{1,2}$, S. Brooks ${ }^{1}$, P. Kubiak-Hardiman ${ }^{1}$, P. Brereton ${ }^{1}$ and M. Dean ${ }^{1}$ \\ ${ }^{1}$ Institute for Global Food Security, Faculty of Medicine, Health and Life Sciences, Queen's University Belfast, \\ Biological Sciences Building, Belfast, UK and \\ ${ }^{2}$ Centre for Public Health, Faculty of Medicine, Health and Life Sciences, Queen's University Belfast, Institute of \\ Clinical Science, Royal Victoria Hospital, Belfast, UK.
}

There is a growing body of evidence to suggest that experiential learning of practical skills related to food, such as gardening or cooking, can play an important role in improving a young person's relationship with food. Food preparation, meal planning and cooking have been shown to be predictors of fruit and vegetable intake and better diet quality ${ }^{(1,2)}$. Furthermore, previous cooking skills interventions with students have shown favourable outcomes in terms of self-efficacy and attitudes towards food ${ }^{(3)}$. Food engagement is a novel concept, which encompasses a multidimensional view of food, from production to waste, the measurement of which may provide a holistic overview of an individual's relationship with food. The aim of this pilot study was to design and deliver a cooking intervention for students, analysing changes in food engagement, as assessed by a validated measure.

Students were recruited from the university setting to participate in a 4-week cooking intervention consisting of four classes, delivered in a cookery school by a trained chef in Nov-Dec 2020. Due to changing COVID-19 restrictions, the latter two sessions took place online. Participants completed a baseline, post-intervention, and a follow-up survey two weeks after completing the intervention. Food engagement scores were calculated for all individuals at each time point.

Participants $(n=13)$ were $53.8 \%$ male with a mean age of 22.8 years; $61.5 \%$ were undergraduate students and $69.2 \%$ were not studying a food-related degree pathway. From baseline to post-intervention, the mean food engagement score increased by $3.0 \%$, which decreased by $0.7 \%$ at follow-up, resulting in an overall $2.3 \%$ increase in mean food engagement score from baseline to follow-up. Participant feedback was overwhelmingly positive: $100 \%(76.9 \%$ strongly) agreed that the cooking classes provided them with new information; $100 \%$ (53.8\% strongly) believed the classes helped them value food more; and, $100 \%(30.8 \%$ strongly) agreed the classes encouraged them to become more engaged with food. Regarding the move online due to COVID-19 restrictions, $100 \%$ (76.9\% strongly) agreed they preferred in-person classes. One participant described the classes as "an amazing experience, where I learned so much and thoroughly enjoyed".

Findings from the pilot study suggest that cooking classes with students may help improve overall food engagement. The pilot was well received and well rated by participants, suggesting acceptability among the student population. Additionally, while students preferred the in-person format, online cooking classes ran successfully, which suggests an online format could be adopted in future to bypass geographical barriers or to reach a larger participant group. Future, larger-scale cooking classes should be conducted within this population to further demonstrate effectiveness and acceptability.

\section{Acknowledgements}

The authors would like to thank Chef Stephen Jeffers of Forestside Cookery School for his collaboration.

\section{References}

1. Hanson AJ, Kattelmann KK, McCormack LA, et al. (2019) Int J Environ Res Public Health 16(14).

2. Larson NI, Perry CL, Story M, et al. (2006) J Am Diet Assoc 106(12), 2001-7.

3. Bernardo GL, Jomori MM, Fernandes AC, et al. (2018) Appetite 130, 247-55. 